\title{
Study of the Characteristics of Solid Phase Crystallized Bridged-Grain Poly-Si TFTs
}

\author{
Wei Zhou, Shuyun Zhao, Rongsheng Chen, Meng Zhang, Jacob Y. L. Ho, \\ Man Wong, Senior Member, IEEE, and Hoi-Sing Kwok, Fellow, IEEE
}

\begin{abstract}
In this paper, bridged-grain (BG) poly-Si thinfilm transistors (TFTs) were fabricated. The characteristics of BG poly-Si TFTs with different BG periods and implantation schemes were investigated. The poly-Si TFTs with optimized BG structures and doping schemes demonstrated greatly improved sub-threshold slope, threshold voltage, maximum field effect mobility, leakage current, and ON/OFF ratio.
\end{abstract}

Index Terms-Bridged grain (BG), polycrystalline silicon, thin-film transistors (TFTs).

\section{INTRODUCTION}

$\mathbf{L}$ OW-TEMPERATURE polycrystalline silicon (LTPS) technology is one of the most promising candidates to realize high resolution active matrix (AM) flat panel displays. Nowadays, ultrahigh pixel density, which brings strict confinement of the size of the thin-film transistors (TFTs) in the pixel, is required by the smart phone and tablet market. Materials with higher mobility, such as polycrystalline silicon (poly-Si) or metal oxide, which allow smaller transistor size, are therefore taking the place of conventional amorphous-Si (a-Si) as the base material for the TFT arrays. For another emerging trend, that for AM organic light emitting diode (AMOLED) display, poly-Si TFTs are also very competitive due to their higher mobility and good stability.

The most widely adopted LTPS technology is laser annealing. Excimer laser annealing [1] and sequential lateral solidification [2] technology produce poly-Si with fewer defects, but at the same time suffer from material nonuniformity due to the random distribution of grain boundaries [3]. Nonuniformity, which will directly result in nonuniform light emission over the whole panel, is highly undesirable for AMOLED displays. Solid phase crystallization (SPC) technology [4] is well-known to be the simplest and most direct method to convert a-Si into

Manuscript received January 8, 2014; revised February 14, 2014 and February 21, 2014; accepted February 24, 2014. Date of publication March 11, 2014; date of current version April 18, 2014. This work was supported by Hong Kong Government Research Grants Council Theme-Based Research Scheme under Grant T23-713/11-1. The review of this paper was arranged by Editor J. Huang.

W. Zhou, R. Chen, M. Zhang, J. Y. L. Ho, M. Wong, and H.-S. Kwok are with the Partner State Key Laboratory, Advanced Displays and Optoelectronics Technologies, Hong Kong University of Science and Technology, Hong Kong (e-mail: eepeggy@ust.hk; eerschen@ust.hk; zhangmeng@ust.hk; eejho@ust.hk; eemwong@ece.ust.hk; eekwok@ust.hk).

$\mathrm{S}$. Zhao is with the Nanoelectronic Fabrication Facility, Hong Kong University of Science and Technology, Hong Kong (e-mail: nfshuyun@ust.hk).

Color versions of one or more of the figures in this paper are available online at http://ieeexplore.ieee.org.

Digital Object Identifier 10.1109/TED.2014.2308579 polycrystalline with small but uniform grains. At the same time, the SPC is also known to be the most time consuming method and produces a high density of defects in the crystallized film, which result in poor TFT performance [5]. With the development of magnetic field-aided SPC technology [6], the crystallization process can be greatly accelerated. But, the TFT performance is still not satisfactory for advanced AM applications. Nanostructures have been adopted into TFTs in various ways to improve the device performance. Poly-Si TFTs based on a trenched body [7] have been proposed to control the back-interface conduction in order to reduce the leakage current. Poly-Si TFTs with 3-D finlike channels [8], [9] were also investigated to enhance the gate controllability over the channel geometry and improve the ON-state current, threshold voltage $\left(V_{\mathrm{th}}\right)$, and subthreshold swing (SS). However, the above methods require fine patterning of $\mathrm{SiO}_{2}$ or poly-Si layers into the nanostructures. Moreover, neither method could significantly improve the ON- and OFF-state characteristics at the same time.

Bridged-grain (BG) technology [10] has been introduced to improve the ON/OFF-current ratio, $V_{\text {th }}, \mathrm{SS}$, and gateinduced drain leakage (GIDL). The benefits of employing a BG structure originate from several aspects. First, in the ON-state, the short channel effects are beneficially employed to reduce the $V_{\text {th }}$ and increase the carrier mobility. Second, the grain size effect: when the grain size is made comparable with the channel length, the BG regions provide shortcuts to the carriers, and help the carriers to and the more conductive path. The number of barriers and traps along the current path is greatly reduced, which would directly result in lower $V_{\text {th }}$, sharper SS, and higher mobility. Third, in the OFF-state, the detrimental effect of a higher leakage current associated with the short channel effects is overcome by the multijunction effects [11] that are inherent in the BG structure due to the reduced electric field near the drain.

For the grain size effect, minimizing the number of grain in the channel can be realized through reducing the length of each subchannel and adopting poly-Si material with larger grain size. For the multijunction effect, adopting larger number of subchannels helps lowering the drain electric field more efficiently. When the channel length on the layout is fixed, using shorter subchannels is a way to enhance the above-mentioned effects. However, as the subchannel length getting scaled, the detrimental aspects of short channel effects exhibited. In short-channel TFTs, the $V_{\text {th }}$ in the linear region decreases due to the reduction of charge in the depletion layer. In the 


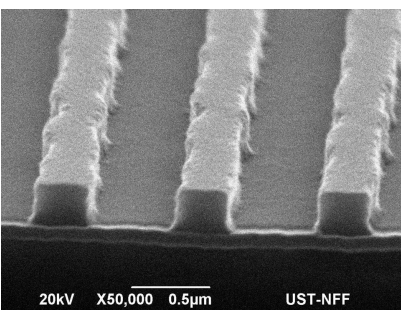

(a)

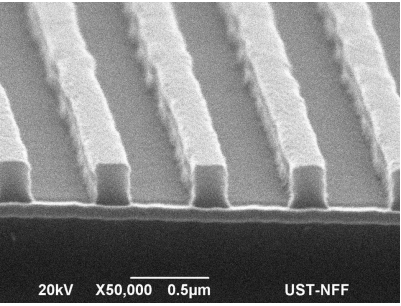

(c)

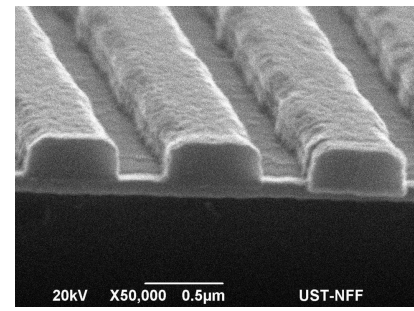

(b)

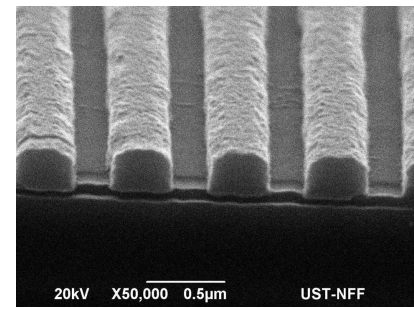

(d)
Fig. 1. SEM images of the patterned photoresist gratings. (a) Positive photoresist $\Lambda=800 \mathrm{~nm}$. (b) Negative photoresist $\Lambda=800 \mathrm{~nm}$. (c) Positive photoresist $\Lambda=600 \mathrm{~nm}$. (d) Negative photoresist $\Lambda=600 \mathrm{~nm}$.

saturation region, drain-induced barrier lowering effect causes an injection of extra carriers and result in larger ON-state current and lower $V_{\text {th }}$. Under high drain bias, punchthrough effect occurs and carriers in the source region can be injected into the depletion region and swept by the field and finally collected at the drain. When punchthrough happens, the SS become worse, leakage current increases. Under significant punchthrough, the gate will lose control over the drain current.

To prevent punchthrough and maximize the BG effect, the BG configuration and doping scheme must be optimized. In this paper, we investigated the device characteristics of the BG TFTs with different BG periods and doping schemes to study the effect of various process parameters on the performance of the BG TFTs. Although laser interference photolithography is applied in this paper to make the grating layer with the period smaller than $1 \mu \mathrm{m}$ as the mask for the BG doping, it should be noted that this grating layer could also be conveniently fabricated over a large area at low cost by adopting nanoimprint technology [12].

\section{DEVICE FABRICATION}

Four-inch sized silicon wafers covered with 500-nm thermal oxide were applied as the starting substrates and 100-nm a-Si was deposited from silane $\left(\mathrm{SiH}_{4}\right)$ onto the substrate through low-pressure chemical vapor deposition (LPCVD) at $550{ }^{\circ} \mathrm{C}$ as the active layer. The SPC process was then carried out through annealing at $600{ }^{\circ} \mathrm{C}$ for $24 \mathrm{~h}$ in $\mathrm{N}_{2}$ to convert the a-Si layer into poly-Si. After crystallization, antireflection coatings (ARC) and photoresist were spin coated. The photoresist layer was then patterned into gratings with different periods $(\Lambda)$ and aspect ratios. Structures of the patterned photoresist were captured by the scanning electron microscope (SEM) and are shown in Fig. 1.

Boron ions were then implanted into the exposed areas through the grating at a dose of $2 \times 10^{15} / \mathrm{cm}^{2}$. To investigate the effect of different implantation schemes, the implantation
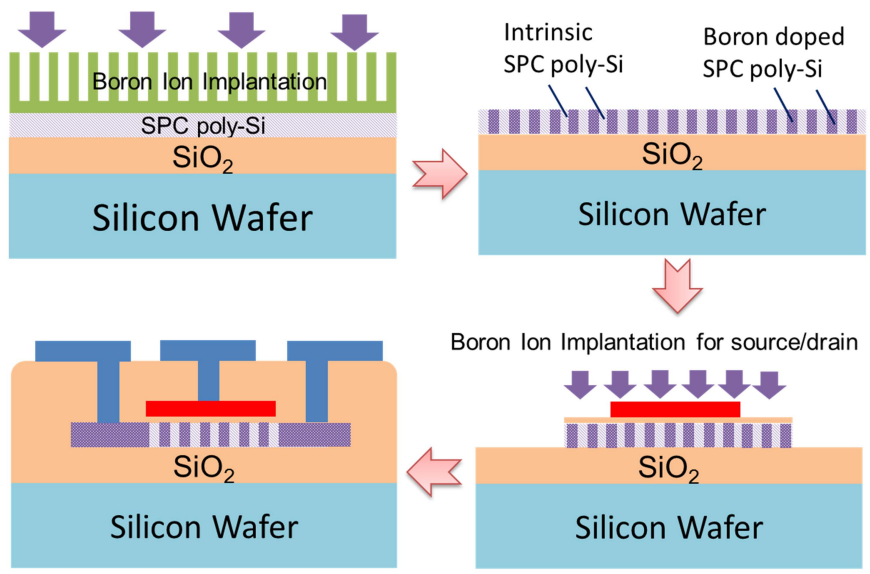

Boron Ion Implantation for source/drain

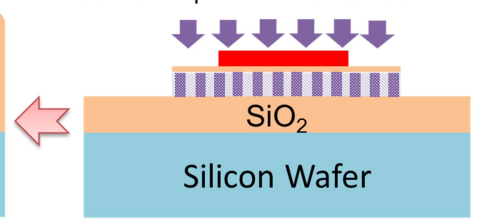

Fig. 2. Key steps of the process flow of BG-SPC TFT fabrication.

TABLE I

Summary OF PROCESS PARAMETERS OF BG-SPC TFTS

\begin{tabular}{cccc}
\hline $\begin{array}{c}\text { Sample } \\
\text { Name }\end{array}$ & $\begin{array}{c}\text { Period } \\
(\mathrm{nm})\end{array}$ & $\begin{array}{c}\text { Aspect Ratio } \\
\text { (Exposed: Covered) }\end{array}$ & $\begin{array}{c}\text { Implantation } \\
\text { Energy(keV) }\end{array}$ \\
\hline $600-13$ & 600 & $2: 1$ & 13 \\
$600-23$ & 600 & $2: 1$ & 23 \\
$600-23 \mathrm{~N}$ & 600 & $1: 2$ & 23 \\
$800-13$ & 800 & $2: 1$ & 13 \\
$800-23$ & 800 & $2: 1$ & 23 \\
$800-23 \mathrm{~N}$ & 800 & $1: 2$ & 23 \\
SPC & No BG & N.A. & N.A \\
\hline \hline
\end{tabular}

energy was varied. After implantation, the photoresist and ARC layer were removed and the poly-Si layer was then patterned into active islands, followed by $70-\mathrm{nm} \mathrm{SiO}_{2}$ deposition through LPCVD at $425{ }^{\circ} \mathrm{C}$ as gate dielectric. Then, 200-nm titanium was sputtered and patterned as gate electrodes and the source and drain were formed through a self-aligned $33-\mathrm{keV}$ boron implantation at a dosage of $4 \times 10^{15} / \mathrm{cm}^{2}$. The contact holes were defined through another 500-nm $\mathrm{SiO}_{2}$ layer, which was adopted as an isolation layer. A 700-nm Al-1\% Si was sputtered and patterned as testing pads. The devices were then sintered in forming gas for $30 \mathrm{~min}$ at $420{ }^{\circ} \mathrm{C}$. No further passivation was applied to these devices. The schematic view of the fabrication process of the BG SPC TFT is shown in Fig. 2.

Table I is given here to summarize the BG periods, exposure fractions, and implantation schemes used in the BG TFTs.

The samples were named in the format of BG periodimplantation energy, and the letter $\mathrm{N}$ was added if the BG lines were formed using a negative photoresist. For example, $600-23 \mathrm{~N}$ represents the BG-TFT with a $600-\mathrm{nm}$ period and negative photoresist patterned BG lines, and the implantation energy used was $23 \mathrm{keV}$.

\section{RESUlTS AND DISCUSSION}

Measurements of device characteristics were done using an HP4156B semiconductor parameter analyzer. The channel width $(W)$ to length $(L)$ ratio of the TFT is $24 / 10 \mu \mathrm{m}$ on the layout. The key parameters of the six types of devices together with the reference SPC TFT without BG are summarized 
TABLE II

Summary of Device Parameters of BG-SPC TFTs

\begin{tabular}{c|ccccccc}
\hline \hline Sample & $\begin{array}{c}600- \\
13\end{array}$ & $\begin{array}{c}600- \\
23\end{array}$ & $\begin{array}{c}600- \\
23 \mathrm{~N}\end{array}$ & $\begin{array}{c}800- \\
13\end{array}$ & $\begin{array}{c}800- \\
23\end{array}$ & $\begin{array}{c}800- \\
23 \mathrm{~N}\end{array}$ & $\mathrm{SPC}$ \\
\hline $\mathrm{L}(\mu \mathrm{m})$ & 10 & 10 & 10 & 10 & 10 & 10 & 10 \\
\hline $\mathrm{L}_{\mathrm{eff}}(\mu \mathrm{m})$ & 3.33 & 3.33 & 6.67 & 3.33 & 3.33 & 6.67 & 10 \\
\hline$V_{t h}(\mathrm{~V})$ & -8 & -4.5 & -10.4 & -11.5 & -9.4 & -117 & -13.8 \\
\hline$S S(\mathrm{~V} / \mathrm{dec})$ & 1.15 & N.A. & 1.06 & 1.17 & 1.1 & 1.17 & 1.72 \\
\hline $\begin{array}{c}\text { on-off ratio } \\
\left(\times 10^{6}\right)\end{array}$ & 15.2 & N.A. & 76.8 & 35 & 53.1 & 53.9 & 1.34 \\
\hline $\mathrm{G}_{\mathrm{m}}(\mathrm{nS})$ & 955 & 1141 & 616 & 785 & 1020 & 558 & 229 \\
\hline $\begin{array}{c}\mu_{\mathrm{FE}} \\
\left(\mathrm{cm}^{2} / \mathrm{Vs}\right)\end{array}$ & 26.1 & N.A. & 33.9 & 21.5 & 28.1 & 30.7 & 19.3 \\
\hline $\begin{array}{c}\mathrm{GIDL} \\
(\mathrm{pA} / \mu \mathrm{m})\end{array}$ & 29.4 & N.A. & 1.89 & 124.5 & 1.43 & 2.58 & 613 \\
\hline \hline
\end{tabular}

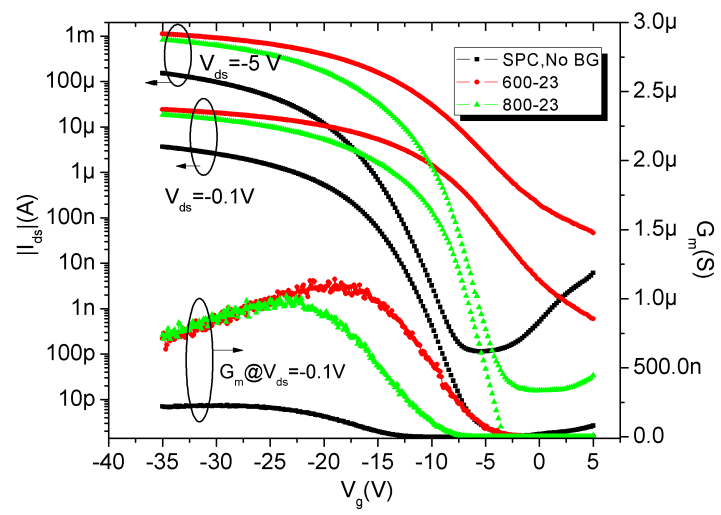

Fig. 3. Transfer characteristics of BG-SPC TFTs with different BG periods.

in Table II. The effect of different BG periods, implantation energy, and aspect ratio are investigated, respectively. Here, the $V_{\text {th }}$ is defined as $V_{g}$ when $\left|I_{d}\right|$ reached $W / L \times 10^{-7} \mathrm{~A}$ at $V_{d}=-5 \mathrm{~V}$. The oN/OFF current ratio is defined as the ratio of maximum and minimum value of $\left|I_{d}\right|$ at $V_{\mathrm{d}}=-5 \mathrm{~V}$. $G_{m}$ stands for the maximum value of transconductance at $V_{d}=-0.1 \mathrm{~V}$. The effective $L$ ( $L_{\text {eff }}$, equals $1 / 3$ or $2 / 3$ of the $L$ on the layout, depending on the aspect ratio of the grating) is used in the calculation of $\mu_{\mathrm{FE}}$. The field effective mobility $\left(\mu_{\mathrm{FE}}\right)$ is estimated using the equation: $\mu_{\mathrm{FE}}=L_{\mathrm{eff}} G_{m} / W C_{\mathrm{ox}} V_{\mathrm{ds}}$, where $C_{\mathrm{ox}}=4.93 \times 10^{-4} \mathrm{~F} \cdot \mathrm{m}^{-2}$.

\section{A. Effect of Changing BG Periods}

For the samples 600-23 and 800-23, the conditions for the BG ion implantation are $23 \mathrm{keV}, 2 \times 10^{15} / \mathrm{cm}^{2}$. From the SEM images of the grating shown in Fig. 1 and the parameters listed in Table I, it is recognized that the effective channel lengths for these samples are almost the same (i.e., 1/3 of the original channel length). The only difference is the period of the BG lines. The effect of the BG period on the device performance is studied through these two samples. The transfer characteristics of BG-TFT 600-23, 800-23 and the reference SPC TFT without BG measured at $V_{\mathrm{ds}}=-0.1 \mathrm{~V}$ and $-5 \mathrm{~V}$ are shown in Fig. 3. From Fig. 3, it can be seen that the
Contours of Boron (6ø0-23) Tilt angle $=\emptyset$

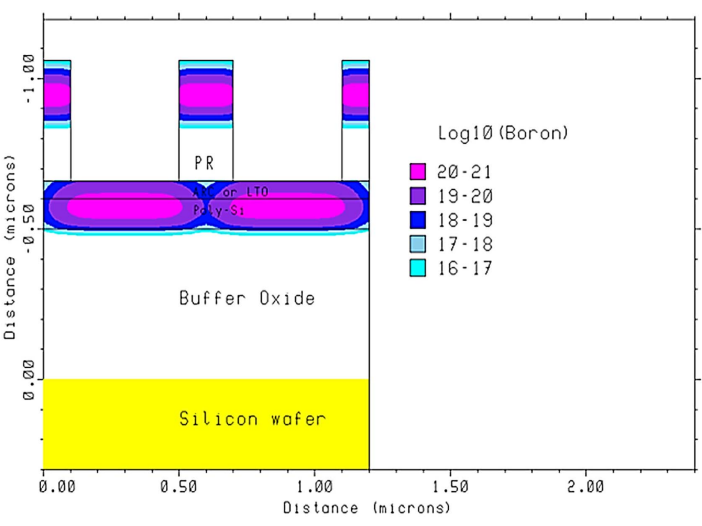

(a)

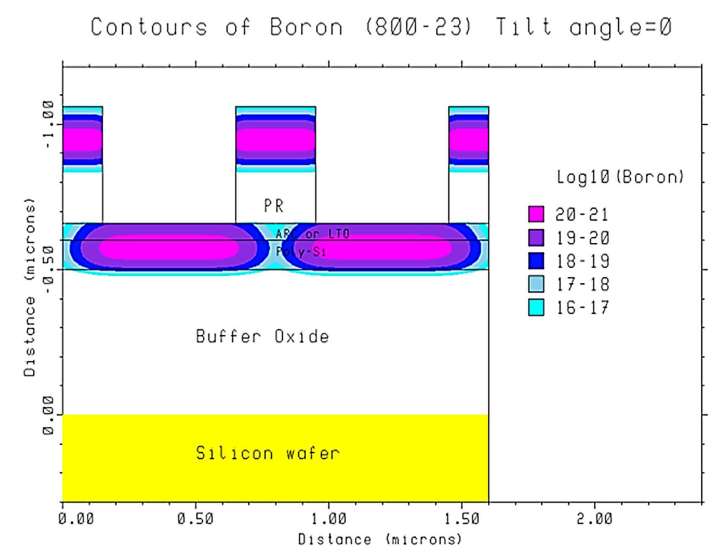

(b)

Fig. 4. Contour plots of dopant distribution for samples with different period of BG lines. (a) 600-23. (b) 800-23.

BG-TFT (sample 800-23) exhibits great reduction in $V_{\text {th }}$, ON/OFF current ratio, $G_{m}$, and SS. These advantages of the BG have been attributed to the grain size effect and shortchannel effect for the enhanced $V_{\mathrm{th}}$, ON-state current and SS, and the drain electric field reduction for the leakage current control. For sample 600-23, although larger ON-state current and $G_{m}$ are observed as we predicted, the OFF-state current is even larger than that of the reference SPC TFT without BG. The poor OFF-state performance of sample 600-23 is due to the lateral scattering of dopants during the BG implantation process.

In an ion implantation process, the collision of the dopant and host is a statistical process. Therefore, for certain implantation energy, the dopant distribution in the host roughly follows a Gaussian function along the depth of the material. The off-axis scattering also results in the lateral expansion of the dopant distribution profile. The projection range and straggle can be simulated using semiconductor process simulation software such as TSUPREM-4. The simulation results are shown in the contour plots shown in Fig. 4(a) and (b) for the two cases discussed above.

It can be seen that for sample 600-23, the small period and lateral scattering result in a wide spread of dopants into the 


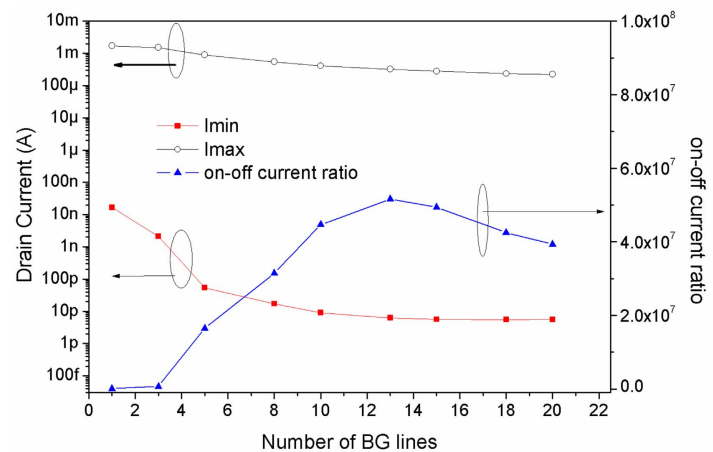

(a)

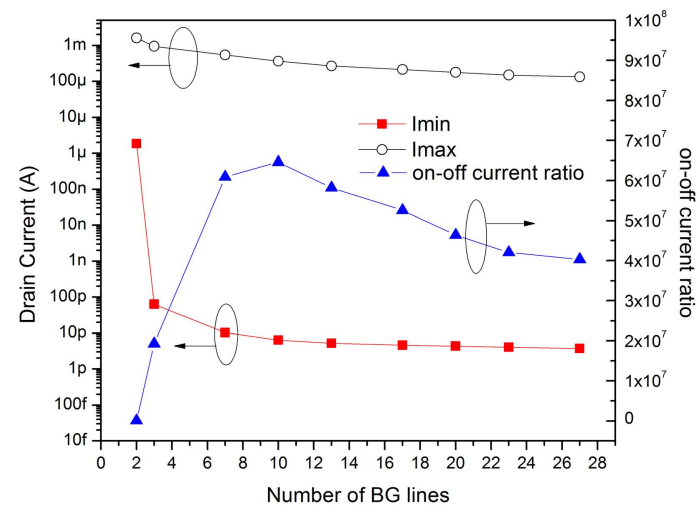

(b)

Fig. 5. Plot of $I_{\max }, I_{\min }$, and ON/OFF current ratio versus number of BG lines. (a) Sample 800-23. (b) Sample 600-23N.

regions protected by the photoresist. The doping concentration in the channel is above $1 \times 10^{18} / \mathrm{cm}^{2}$, which makes the short channels start to be conductive. As a result, although the ON-state current for sample $600-23$ is larger, the TFT cannot be turned OFF. For sample 800-23, the channel dopant concentration is more than one order lower than 600-23. From Fig. 3, it can be seen that sample 800-23 works properly, with obvious improvement of device characteristics compared with the device without BG.

For sample $800-23$ and $600-23 \mathrm{~N}$, the relationship between the number of BG lines and the maximum/minimum drain current ( $I_{\max }$ and $I_{\min }$, respectively) and ON/OFF current ratio are plotted in Fig. 5(a) and (b), respectively. The channel length on the layout is varied from 1 to $16 \mu \mathrm{m}$, resulting in 1-20 BG regions in the channel for $800-23$, or 2-27 BG regions for $600-23 \mathrm{~N}$. The width of the channel is fixed to be equal to $10 \mu \mathrm{m}$.

Fig. 5 shows that $I_{\max }$ decreases for both samples with a larger number of BG lines, which is due to the longer effective channel length. Sample 800-23 shows a larger ON-state current due to shorter effective channel length. $I_{\min }$ also decreases with a larger number of BG lines, but the decrease of $I_{\min }$ is not only due to the channel length variation, but is also due to the drain electric field lowering effect. Therefore, the ON/OFF ratio is not simply a constant for different channel lengths, as shown in the blue curve in Fig. 5(a) and (b). The effect of the drain electric field lowering is saturated when the

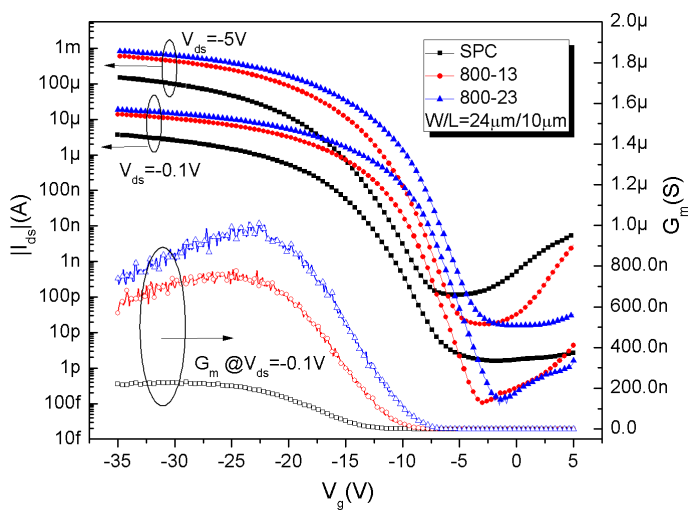

(a)

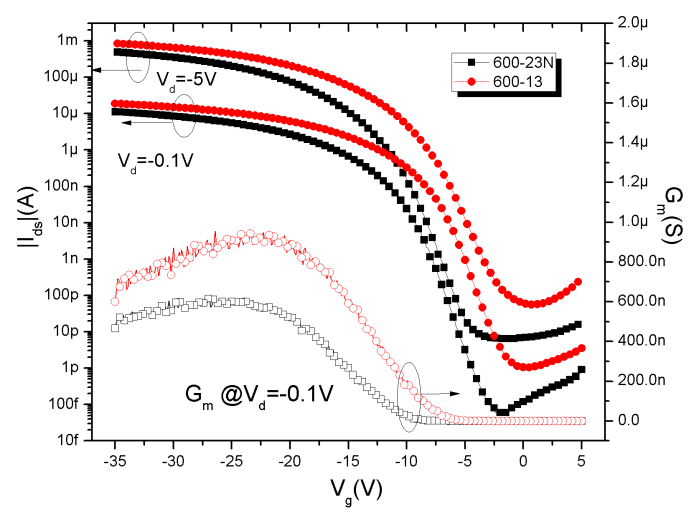

(b)

Fig. 6. Transfer characteristics of BG-SPC TFTs with different implantation energies. (a) $800-13$ and $800-23$. (b) $600-13$ and $600-23 \mathrm{~N}$.

number of BG lines is large enough. Further increasing the number of BG lines shows no benefit to the ON/OFF current ratio due to the reduced ON-state current. The ON/OFF ratio already reaches $1 \times 10^{7}$ when there are five lines (i.e., $4-\mu \mathrm{m}$ channel length) in the channel for 800-23, and it reaches a maximum value of above $5 \times 10^{7}$ when there are 13 lines (i.e., $10-\mu \mathrm{m}$ channel length). Saturation of current ON/OFF current ratio happens at a smaller BG number for sample $600-23 \mathrm{~N}$, due to the longer subchannel length $(\sim 400 \mathrm{~nm}$ for each subchannel) and less short channel effect than in sample $800-23$. With $6-\mu \mathrm{m}$ channel length on the layout (10 BG lines in the channel), the ON/OFF ratio can be as high as $6 \times 10^{7}$. Therefore, when the channel length is fixed, it is preferred to have a shorter BG period in order to introduce more BG lines in the channel. However, the separation between neighboring short channels must be properly chosen, with careful consideration of the lateral distribution of dopants, as discussed above.

\section{B. Effect of Changing Ion Implantation Energy}

For the samples 800-13 and 800-23, the conditions for the BG ion implantation energy are 13 and $23 \mathrm{keV}$, respectively, with the same dose of $2 \times 10^{15} / \mathrm{cm}^{2}$. The number of BG lines in the channel and the effective channel lengths are the same. The transfer characteristics of BG-TFT 800-13, 800-23 and the reference SPC TFT measured at $V_{\mathrm{ds}}=-0.1$ and $-5 \mathrm{~V}$ are shown in Fig. 6(a). 
Contours of Boron (800-13) Tilt angle $=\emptyset$

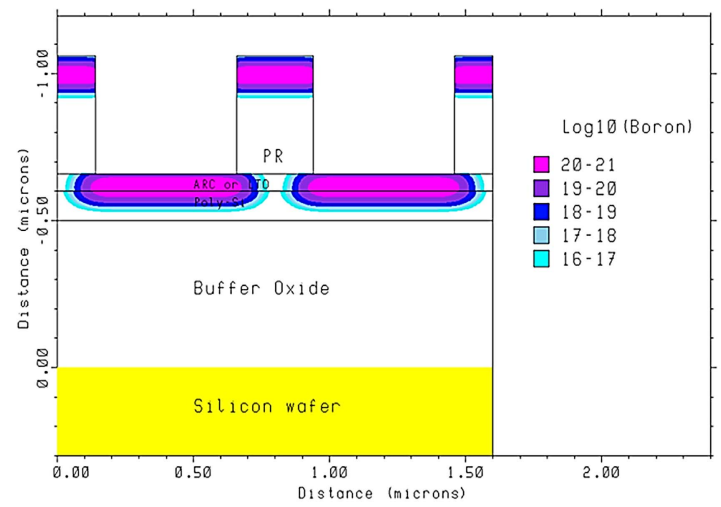

Fig. 7. Contour plots of dopant distribution for sample 800-13.

It can be observed that $800-13$ shows much less reduction in $V_{\text {th }}$ and GIDL than 800-23. However, the SS, maximum ON-state current and minimum leakage current of these two samples are comparable. The boron dopant distribution profiles are simulated and shown in Fig. 7. It is obvious that higher implantation energy will result in a deeper location of the peak of the dopant concentration. At the same time, the lateral scattering profile is also wider with higher implantation energy. The shorter effective channel length and lightly doped channel result in a larger ON-state current and lower $V_{\text {th }}$ for 800-23. For 800-13, a lot of the dopants are not implanted into the channel, but remain in the ARC layer, and the channel is only doped for the upper half. To confirm the reason for the insufficient leakage current suppression, the transfer characteristics of $600-13$ and $600-23 \mathrm{~N}$ are also compared. As shown in Fig. 6(b), for 600-13, the GIDL leakage current is larger than $600-23 \mathrm{~N}$, despite the effective channel length of $600-13$ being shorter than $600-23 \mathrm{~N}$, which results in a larger ON-state current and smaller $V_{\text {th }}$. This result indicates that the poor GIDL control in 600-13 is due to shallow implantation rather than the BG period or effective channel length, which suggests that the back-interface conduction [13] is not well controlled. Therefore, it is necessary to adjust the implantation energy to fully cover the depth of the channel. As the implantation energy also affects the lateral scattering profile, to prevent a lateral short circuit of the BG lines and get enough implantation depth at the same time, the BG period and thickness of the related layers must be carefully designed.

\section{Effect of the Aspect Ratio}

The exposure ratio in one period also affects the performance of BG-SPC TFTs. The transfer characteristics of $600-23 / 600-23 \mathrm{~N}$ and $80-23 / 80-23 \mathrm{~N}$ are shown in Fig. 8(a) and (b), respectively. For 600-nm period BG lines, the exposure ratio in one period plays a very important role. As previously discussed and shown, for small period BG lines, the lateral scattering may result in a short circuit of the BG lines. The poor ON/OFF ratio of sample 600-23 is caused by the lateral scattering of dopants. The advantages of using BG are clearly observed in $600-23 \mathrm{~N}$, although the ON-state current is not as large as $600-23$ due to the longer effective

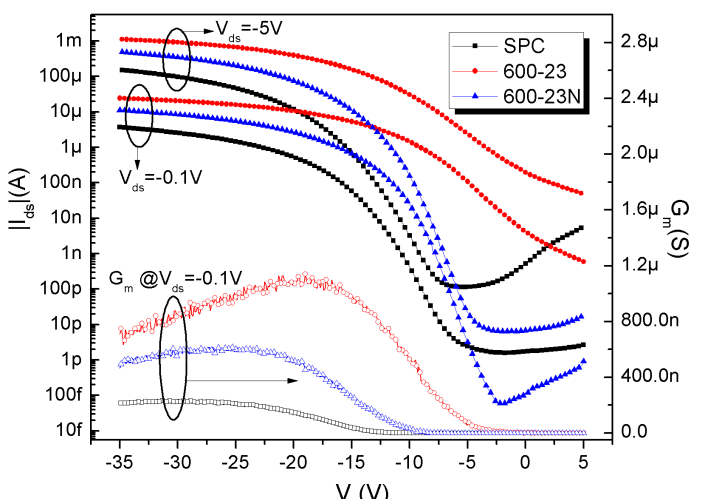

(a)

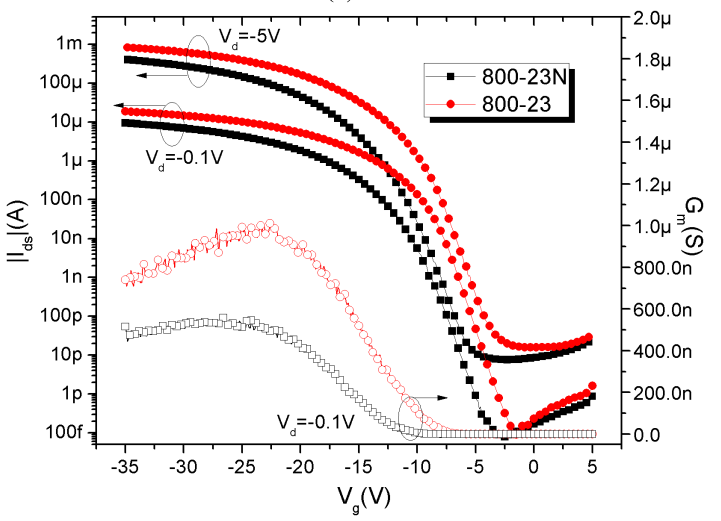

(b)

Fig. 8. Transfer characteristics of BG-SPC TFTs with different aspect ratio. (a) $600-23$ and $600-23 \mathrm{~N}$ and (b) $800-23$ and $800-23 \mathrm{~N}$.

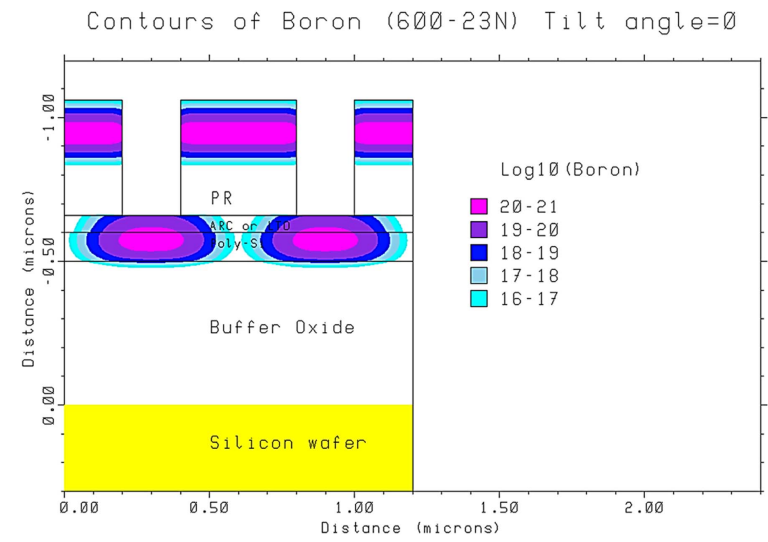

Fig. 9. Contour plots of dopant distribution for sample $600-23 \mathrm{~N}$.

channel length. The simulation result of the dopant distribution for $600-23 \mathrm{~N}$ is shown in Fig. 9. Compared with the previous plot of 600-23, shown in Fig. 4(a), partially lightly doped short channels can be formed in 600-23N. As shown in Fig. 8(b), samples $800-23$ and $800-23 \mathrm{~N}$ can both work properly with BG structures, which indicate that the lateral scattering of dopants is not the critical parameter for $800-\mathrm{nm}$ period BG lines at an implantation energy of $23 \mathrm{keV}$. Of course, 800-23 demonstrates larger ON-state current and lower $V_{\text {th }}$ due to the shorter effective channel length. The GIDL currents for 800-23 and $800-23 \mathrm{~N}$ are comparable, as the implantation depths of the BG dopants are the same for these two samples. 


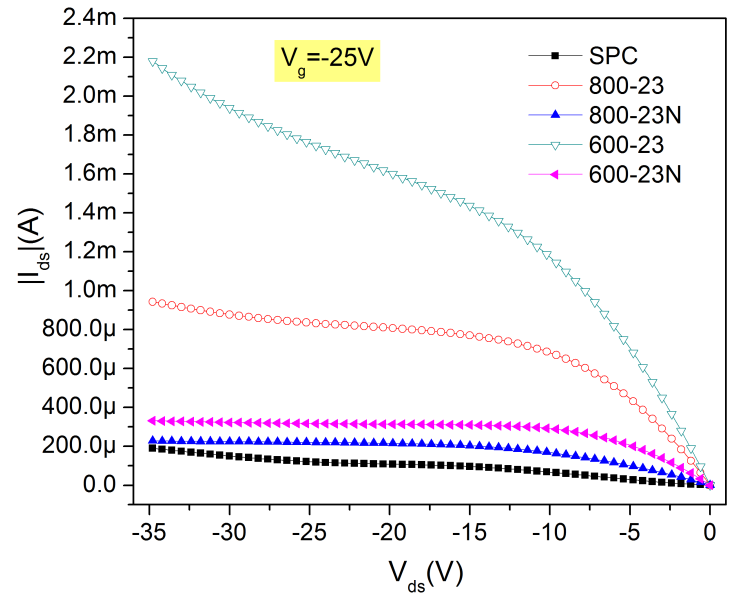

Fig. 10. Output characteristics of the SPC and BG-SPC TFTs.

The output characteristics of the BG TFTs using the same implantation condition but different BG configurations measured at $V_{g}=-25 \mathrm{~V}$ is plotted in Fig. 10, together with the output characteristics of the reference SPC TFT ( $W / L=24 / 10 \mu \mathrm{m}$ on the layout). The kink effect is related to the drain electric field. The main cause of the kink effect is impact ionization near the drain at high drain voltage. This effect is also observed in Silicon On Insulator (SoI) devices due to the same floating body in TFTs and SoI transistors. However, in poly-Si TFTs, the carriers trapped at the grain boundaries can be freed under the high drain electric field, which makes the situation much more serious.

From Fig. 10, kink effect is observed in the reference SPC TFTs (see the black curve with square marks). Short-channel effects result in enhanced kink effect. However, the inherent multijunction structure in a BG TFT helps reducing the electric field near the drain depletion region and relieving the kink effect. Although the subchannel lengths of the BG TFTs are in submicrometer scale, for sample $600-23 \mathrm{~N}, 800-23 \mathrm{~N}$, and 800-23, the kink effect is still sufficiently suppressed. This improvement coincides with the GIDL reduction observed in the transfer characteristics of these BG TFTs. For sample 600-23, the enhanced kink effect is observed and it is due to the over-scaled subchannel length and ion scattering during the implantation process. This observation is also in accordance with the transfer characteristics of 600-23 shown in Fig. 3.

\section{CONCLUSION}

The BG SPC TFTs were fabricated and characterized. Larger ON-state current and ON/OFF current ratio, lower $V_{\text {th }}$, and sharper SS were achieved in BG TFTs, making use of the merits of the grain size effect, short channel effect, and drain electric field relief. The influence of different process parameters on the device performance was investigated. It was shown that the period of the $\mathrm{BG}$ lines, exposure ratio, and the BG implantation energy all affect the performance of BG TFTs from different aspects. The BG period is expected to be made small to increase the number of BG lines inside the channel as long as the BG lines are not shorted through lateral scattering. The optimized BG SPC TFTs are promising for advanced AM applications.

\section{REFERENCES}

[1] T. Sameshima, S. Usui, and M. Sekiya, "XeCl excimer laser annealing used in the fabrication of poly-Si TFT's," IEEE Electron Device Lett., vol. 7, no. 5, pp. 276-278, May 1986.

[2] R. Sposili and J. Im, "Sequential lateral solidification of thin silicon films on $\mathrm{SiO}_{2}$," Appl. Phys. Lett., vol. 69, pp. 2864-2866, Nov. 1996.

[3] J. B. Choi et al., "Sequential lateral solidification (SLS) process for large area AMOLED," in SID Symp., Dig. Tech. Papers, vol. 39. 2008, pp. 97-100.

[4] M. Hatalis and D. Greve, "High-performance thin-film transistors in low-temperature crystallized LPCVD amorphous-silicon films," IEEE Electron Device Lett., vol. 8, no. 8, pp. 361-364, Aug. 1987.

[5] Y. Morimoto, Y. Jinno, K. Hirai, H. Ogata, T. Yamada, and K. Yoneda, "Influence of the grain boundaries and intragrain defects on the performance of poly-Si thin film transistors," J. Electrochem. Soc., vol. 144, pp. 2495-2501, Jul. 1997.

[6] H. Seo et al., "Alternating magnetic field-assisted crystallization of Si films without metal catalyst," J. Cryst. Growth, vol. 310, pp. 5317-5320, Dec. 2008.

[7] J. Lin and K. Huang, "A high-performance polysilicon thin-film transistor built on a trenched body," IEEE Trans. Electron Devices, vol. 55, no. 9, pp. 2417-2422, Sep. 2008.

[8] H. J. H. Chen, J. Jhang, C. Huang, S. Chen, and J. Huang, "Poly-Si TFTs with three-dimensional finlike channels fabricated using nanoimprint technology," IEEE Electron Device Lett., vol. 32, no. 2, pp. 155-157, Feb. 2011.

[9] H. J. H. Chen, J. Jhang, and C. Huang, "Study on characteristics of poly-Si TFTs with 3-D finlike channels fabricated by nanoimprint technology," IEEE Trans. Electron Devices, vol. 59, no. 9, pp. 2314-2320, Sep. 2012.

[10] W. Zhou et al., "Bridged-grain solid-phase-crystallized polycrystallinesilicon thin-film transistors," IEEE Electron Device Lett., vol. 33, no. 10 pp. 1414-1416, Oct. 2012.

[11] Y. Uemoto, E. Fujii, F. Emoto, A. Nakamura, and K. Senda, "A highvoltage polysilicon TFT with multigate structures," IEEE Trans. Electron Devices, vol. 38, no. 1, pp. 95-100, Jan. 1991.

[12] W. Zhou, J. Y. L. Ho, S. Zhao, R. Chen, M. Wong, and H. Kwok, "Fabrication of bridged-grain polycrystalline silicon thin film transistors by nanoimprint lithography," Thin Solid Films, vol. 534, pp. 636-639, May 2013.

[13] S. Brotherton, J. Ayres, and M. Trainor, "Control and analysis of leakage currents in poly-Si thin-film transistors," J. Appl. Phys., vol. 79, pp. 895-904, Jan. 1996.

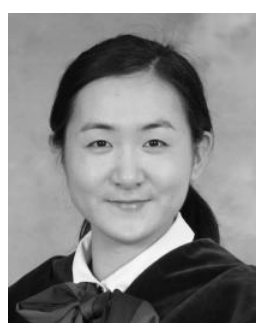

Wei Zhou received the Ph.D. degree from the Department of Electronic and Computer Engineering, Hong Kong University of Science and Technology, Hong Kong, in 2013.

Her current research interests include lowtemperature poly-Si thin-film transistors (TFTs), novel oxide TFTs, and their application in displays.

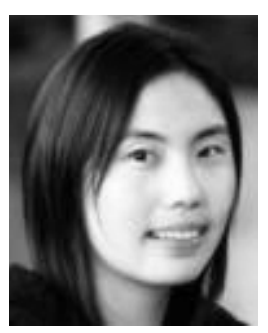

Shuyun Zhao received the Ph.D. degree from the Department of Electronic and Computer Engineering, Hong Kong University of Science and Technology, Hong Kong, in 2011.

Her current research interests include the development of low-temperature polycrystalline silicon technology for display applications. 


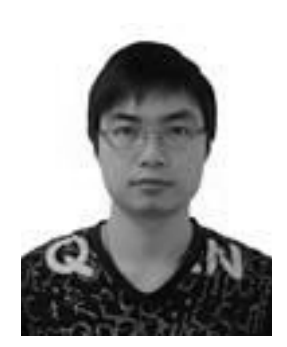

Rongsheng Chen received the Ph.D. degree from the Department of Electronic and Computer Engineering, Hong Kong University of Science and Technology, Hong Kong, in 2013.

His current research interests include novel compound thin-film transistors based on $\mathrm{ZnO}$, IGZO, and $\mathrm{GaN}$, and their application in active matrix displays.

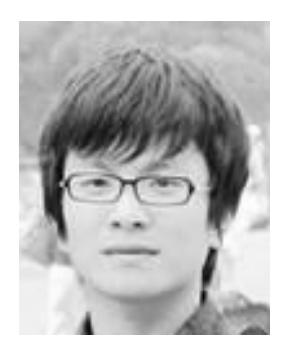

Meng Zhang is currently pursuing the Ph.D. degree with the Department of Electronic and Computer Engineering, Hong Kong University of Science and Technology, Hong Kong.

His current research interests include novel thinfilm transistor (TFT) structures, oxide TFTs, and reliability of poly-Si TFTs.

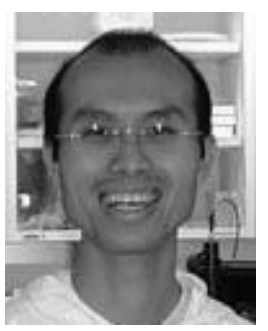

Jacob Y. L. Ho received the M.S. degree in electrical and electronic engineering from Hong Kong University of Science and Technology (HKUST), Hong Kong, in 1999.

He is currently a Senior Technician with HKUST.

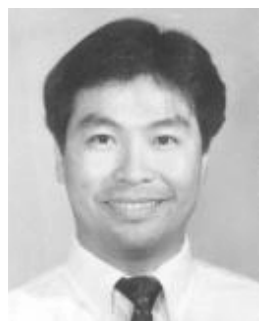

Man Wong (M'84-SM'00) received the Ph.D. degree in electrical engineering from the Center for Integrated Systems, Stanford University, Palo Alto, CA, USA.

He joined the faculty of the Department of Electrical and Electronic Engineering, Hong Kong University of Science and Technology, Hong Kong, in 1992.

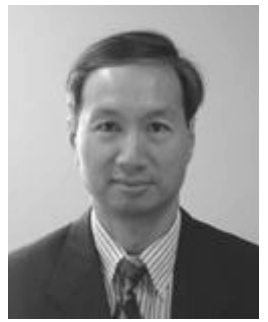

Hoi-Sing Kwok (M'78-SM'84-F'05) received the Ph.D. degree in applied physics from Harvard University, Cambridge, MA, USA, in 1978.

He joined Hong Kong University of Science and Technology, Hong Kong, in 1992, where he is currently the Director with the Center for Display Research and the Director with the Partner State Key Laboratory on Advanced Displays and Optoelectronics Technologies. 